\title{
THE CONTRACTION PRINCIPLE FOR MAPPINGS ON A METRIC SPACE WITH A GRAPH
}

\author{
JACEK JACHYMSKI \\ (Communicated by Joseph A. Ball) \\ To the memory of Professor Andrzej Lasota*
}

\begin{abstract}
We give some generalizations of the Banach Contraction Principle to mappings on a metric space endowed with a graph. This extends and subsumes many recent results of other authors which were obtained for mappings on a partially ordered metric space. As an application, we present a theorem on the convergence of successive approximations for some linear operators on a Banach space. In particular, the last result easily yields the Kelisky-Rivlin theorem on iterates of the Bernstein operators on the space $C[0,1]$.
\end{abstract}

\section{INTRODUCTION}

Let $f$ be a selfmap of a metric space $(X, d)$. Following Petruşel and Rus [PR06], we say that $f$ is a Picard operator (abbr., PO) if $f$ has a unique fixed point $x_{*}$ and $\lim _{n \rightarrow \infty} f^{n} x=x_{*}$ for all $x \in X$. Recently, many results appeared giving sufficient conditions for $f$ to be a PO if $(X, d)$ is endowed with a partial ordering $\preceq$. Most of them are a hybrid of two fundamental fixed point theorems: the Banach Contraction Principle and the Knaster-Tarski theorem (see, e.g., [GD03, p. 25] and, for a discussion on its applications in metric fixed point theory, [Ja01]). Indeed, they deal with a monotone (either order-preserving or order-reversing) mapping satisfying, with some restriction, a classical contractive condition, and such that for some $x_{0} \in X$, either $x_{0} \preceq f x_{0}$ or $f x_{0} \preceq x_{0}$. The first result in this direction was given by Ran and Reurings [RR04] who also presented its applications to linear and nonlinear matrix equations.

Theorem 1.1 ([RR04], Th. 2.1). Let $(X, d)$ be a complete metric space endowed with a partial ordering $\preceq$ such that

every pair of elements of $X$ has an upper and a lower bound.

Let $f: X \rightarrow X$ be continuous and monotone, and such that

$$
\exists_{\alpha \in(0,1)} \forall_{x, y \in X}(x \preceq y \Rightarrow d(f x, f y) \leq \alpha d(x, y)) .
$$

If there exists $x_{0} \in X$ with $x_{0} \preceq f x_{0}$ or $f x_{0} \preceq x_{0}$, then $f$ is a PO.

Received by the editors December 12, 2006 and, in revised form, February 13, 2007.

2000 Mathematics Subject Classification. Primary 47H10; Secondary 05C40, 54H25.

Key words and phrases. Fixed point, Picard operator, partial order, connected graph, Bernstein operator.

* Professor Andrzej Lasota passed away on December 28, 2006. 
Subsequently, Nieto and Rodríguez-López [NR-L05] extended Theorem 1.1 in the following way.

Theorem 1.2 ([NR-L05], Ths. 2.1-2.5). Let $(X, d)$ be a complete metric space endowed with a partial ordering $\preceq$. Let $f: X \rightarrow X$ be nondecreasing (order-preserving) and such that (1.2) holds. Assume that one of the the following conditions holds:

(A) $f$ is continuous and there exists $x_{0} \in X$ with $x_{0} \preceq f x_{0}$ or $f x_{0} \preceq x_{0}$;

(B) $(X, d, \preceq)$ is such that

for any nondecreasing $\left(x_{n}\right)_{n \in \mathbb{N}}$, if $x_{n} \rightarrow x$, then $x_{n} \preceq x$ for $n \in \mathbb{N}$, and there exists $x_{0} \in X$ with $x_{0} \preceq f x_{0}$;

(C) $(X, d, \preceq)$ is such that

for any nonincreasing $\left(x_{n}\right)_{n \in \mathbb{N}}$, if $x_{n} \rightarrow x$, then $x \preceq x_{n}$ for $n \in \mathbb{N}$, and there exists $x_{0} \in X$ with $f x_{0} \preceq x_{0}$.

Then $f$ has a fixed point. Moreover, if $(X, \preceq)$ is such that every pair of elements of $X$ has an upper or a lower bound, then $f$ is a PO.

As an application, the authors obtained a theorem on the existence of a unique solution for periodic boundary problems relative to ordinary differential equations. Similar applications based on a version of Theorem 1.2 for a mixed monotone mapping $F: X \times X \rightarrow X$ were given by Gnana Bhaskar and Lakshmikantham [G-BL06].

Further improvements of the above results were found independently by Petrusel and Rus [PR06], and Nieto and Rodríguez-López [NR-L07]. Here we give a slightly more general version of these extensions taken from the paper by Nieto, Pouso and Rodríguez-López [NPR-L07]. Following [PR06] we denote:

$$
X_{\preceq}:=\{(x, y) \in X \times X: x \preceq y \text { or } y \preceq x\} .
$$

Theorem 1.3 ([PR06], Th. 4.3; [NR-L07], Th. 7; [NPR-L07], Th. 3.1). Let $(X, d)$ be a complete metric space endowed with a partial ordering $\preceq$ such that (1.5) holds. Let $f: X \rightarrow X$ be such that $f$ preserves comparable elements, i.e.,

$$
\text { for any } x, y \in X,(x, y) \in X_{\preceq} \text { implies }(f x, f y) \in X_{\preceq},
$$

and (1.2) holds. Assume that either $f$ is orbitally continuous (cf. Definition 2.2) or $(X, d, \preceq)$ is such that

$$
\begin{aligned}
& \text { for any }\left(x_{n}\right)_{n \in \mathbb{N}} \text {, if } x_{n} \rightarrow x \text { and }\left(x_{n}, x_{n+1}\right) \in X_{\preceq} \text { for } n \in \mathbb{N} \text {, then } \\
& \text { there is a subsequence }\left(x_{k_{n}}\right)_{n \in \mathbb{N}} \text { such that }\left(x_{k_{n}}, x\right) \in X_{\preceq} \text { for } n \in \mathbb{N} \text {. }
\end{aligned}
$$

If there exists $x_{0} \in X$ with $\left(x_{0}, f x_{0}\right) \in X_{\preceq}$, then $f$ is a PO.

(Actually, in [PR06] (1.1) was used instead of (1.5), and (1.7) was not considered; moreover, the authors required the compatibility between a metric and an order structure (cf. [PR06, Def. 2.1, (iii) and (iv)]) which, however, was not necessary in Theorem 1.3.)

Our purpose here is twofold: first, we want to establish results which generalize and subsume the above theorems; second, we wish to present some applications to the theory of linear operators. Regarding the first aim, it seems it is more convenient here to use the language of graph theory instead of partial orderings. So we 
are going to study the class of generalized Banach contractions on a metric space endowed with a directed graph. One of the advantages is that, for example, conditions (1.3), (1.4) and (1.7) can be described then in a unified way. Moreover, (1.5) may be significantly weakened and replaced by a natural condition of connectivity of some graph as shown in Section 3. In fact, this connectivity turns out to be a necessary and sufficient condition for any generalized Banach contraction to be a PO (cf. Corollaries 3.2 and 3.3). If a graph is disconnected, then such a contraction may have many fixed points. In this case we give a characterization of cardinality of the set of fixed points, and we provide more exact information on the convergence of successive approximations (cf. Theorem 3.2). Furthermore, Theorem 3.4 shows that for an orbitally continuous mapping $f$ the assumption 'there exists $x_{0} \in X$ with $x_{0} \preceq f x_{0}$ or $f x_{0} \preceq x_{0}$ ' occurring in all the above theorems is superfluous (in particular, it can be dropped in Theorem 1.1) though, without a continuity condition, it cannot be removed. Also, Theorem 3.4 yields the well-known fixed point theorem of Edelstein [E61] for uniformly locally contractive mappings (cf. Corollary 3.4).

Finally, as an application of our results, we give a sufficient condition for a linear operator $T$ on a Banach space $X$ to be a weakly Picard operator (abbr., WPO), i.e., for any $x \in X, \lim _{n \rightarrow \infty} T^{n} x$ exists (it may depend on $x$ ) and is a fixed point of $T$. As a simple consequence, we get the Kelisky-Rivlin [KR67] theorem on iterates of the classical Bernstein operators on the Banach space $C[0,1]$.

\section{BASIC CONCEPTS AND NOTATIONS}

Let $(X, d)$ be a metric space. Let $\Delta$ denote the diagonal of the Cartesian product $X \times X$. Consider a directed graph $G$ such that the set $V(G)$ of its vertices coincides with $X$, and the set $E(G)$ of its edges contains all loops, i.e., $E(G) \supseteq \Delta$. (Example 2.1 illuminates a need of making the latter assumption.) We assume $G$ has no parallel edges, so we can identify $G$ with the pair $(V(G), E(G))$. Moreover, we may treat $G$ as a weighted graph (see [Jo97, p. 309]) by assigning to each edge the distance between its vertices.

By $G^{-1}$ we denote the conversion of a graph $G$, i.e., the graph obtained from $G$ by reversing the direction of edges. Thus we have

$$
E\left(G^{-1}\right)=\{(x, y) \in X \times X:(y, x) \in E(G)\} .
$$

The letter $\tilde{G}$ denotes the undirected graph obtained from $G$ by ignoring the direction of edges. Actually, it will be more convenient for us to treat $\tilde{G}$ as a directed graph for which the set of its edges is symmetric. Under this convention,

$$
E(\tilde{G})=E(G) \cup E\left(G^{-1}\right) .
$$

We call $\left(V^{\prime}, E^{\prime}\right)$ a subgraph of $G$ if $V^{\prime} \subseteq V(G), E^{\prime} \subseteq E(G)$ and for any edge $(x, y) \in E^{\prime}, x, y \in V^{\prime}$.

Definition 2.1. We say that a mapping $f: X \rightarrow X$ is a Banach $G$-contraction or simply $G$-contraction if $f$ preserves edges of $G$, i.e.,

$$
\forall_{x, y \in X}((x, y) \in E(G) \Rightarrow(f x, f y) \in E(G)),
$$

and $f$ decreases weights of edges of $G$ in the following way:

$$
\exists_{\alpha \in(0,1)} \forall_{x, y \in X}((x, y) \in E(G) \Rightarrow d(f x, f y)<\leq \alpha d(x, y)) .
$$

Remark 2.1. It is easy to observe that $(2.2)$ means $(f(V(G)),(f \times f)(E(G)))$ is a subgraph of $G$, where $(f \times f)(x, y):=(f x, f y)$ for $x, y \in X$. 
Example 2.1. Any constant function $f: X \rightarrow X$ is a Banach $G$-contraction since $E(G)$ contains all loops. (In fact, $E(G)$ must contain all loops if we wish any constant function to be a $G$-contraction.)

Example 2.2. Any Banach contraction is a $G_{0}$-contraction, where the graph $G_{0}$ is defined by $E\left(G_{0}\right):=X \times X$.

Example 2.3. Let $\preceq$ be a partial order in $X$. Define the graph $G_{1}$ by

$$
E\left(G_{1}\right):=\{(x, y) \in X \times X: x \preceq y\} .
$$

For this graph, (2.2) means $f$ is nondecreasing with respect to this order. Recently, the class of $G_{1}$-contractions was studied by Nieto and Rodríguez-López [NR-L05].

The next example will be preceded by the following

Proposition 2.1. If a mapping $f: X \rightarrow X$ is such that (2.2) (resp., (2.3)) holds, then (2.2) (resp., (2.3)) is also satisfied for graphs $G^{-1}$ and $\tilde{G}$. Hence, if $f$ is a $G$-contraction, then $f$ is both a $G^{-1}$-contraction and a $\tilde{G}$-contraction.

Proof. This is an obvious consequence of symmetry of $d$ and (2.1).

Example 2.4. Let $\preceq$ be a partial order in $X$. Set

$$
E\left(G_{2}\right):=\{(x, y) \in X \times X: x \preceq y \vee y \preceq x\} .
$$

In particular, for this graph (2.2) holds if $f$ is monotone with respect to the order. Moreover, if $f$ satisfies (2.3) with $G:=G_{1}$ from Example 2.3, then by Proposition 2.1, (2.3) holds with $G:=G_{2}$ since $G_{2}=\tilde{G}_{1}$. Hence mappings studied by Ran and Reurings [RR04] are $G_{2}$-contractions. In general, (2.2) with $G:=G_{2}$ means $f$ maps comparable elements onto comparable elements, so the class of $G_{2^{-}}$ contractions coincides with the class considered by Petruşel and Rus [PR06], and Nieto and Rodríguez-López [NR-L07].

Now we discuss some types of continuity of mappings. The first of them is well known and often used in metric fixed point theory (see, e.g., [PR06]).

Definition 2.2. A mapping $f: X \rightarrow X$ is called orbitally continuous if for all $x, y \in X$ and any sequence $\left(k_{n}\right)_{n \in \mathbb{N}}$ of positive integers,

$$
f^{k_{n}} x \rightarrow y \text { implies } f\left(f^{k_{n}} x\right) \rightarrow f y \text { as } n \rightarrow \infty \text {. }
$$

Definition 2.3. A mapping $f: X \rightarrow X$ is called $G$-continuous if given $x \in X$ and a sequence $\left(x_{n}\right)_{n \in \mathbb{N}}$,

$$
x_{n} \rightarrow x \text { and }\left(x_{n}, x_{n+1}\right) \in E(G) \text { for } n \in \mathbb{N} \text { imply } f x_{n} \rightarrow f x .
$$

In particular, $G_{1}$-continuity (cf. Example 2.3) means $f$ maps convergent and nondecreasing sequences onto convergent sequences. If $X:=\mathbb{R}$ is endowed with the Euclidean metric, then $f$ is $G_{1}$-continuous iff $f$ is left continuous. Another example concerning $G$-continuity will be given in Section 4 .

The next definition is inspired by the concept of orbital monotone-continuity introduced in [NPR-L07, Th. 4.1].

Definition 2.4. A mapping $f: X \rightarrow X$ is called orbitally $G$-continuous if for all $x, y \in X$ and any sequence $\left(k_{n}\right)_{n \in \mathbb{N}}$ of positive integers,

$$
f^{k_{n}} x \rightarrow y \text { and }\left(f^{k_{n}} x, f^{k_{n+1}} x\right) \in E(G) \text { for } n \in \mathbb{N} \text { imply } f\left(f^{k_{n}} x\right) \rightarrow f y .
$$


Clearly, we have the following relations:

continuity $\Rightarrow$ orbital continuity $\Rightarrow$ orbital $G$-continuity;

continuity $\Rightarrow G$-continuity $\Rightarrow$ orbital $G$-continuity.

Now we recall a few basic notions concerning connectivity of graphs. All of them can be found, e.g., in [Jo97, Ch. 6]. If $x$ and $y$ are vertices in a graph $G$, then a path in $G$ from $x$ to $y$ of length $N(N \in \mathbb{N} \cup\{0\})$ is a sequence $\left(x_{i}\right)_{i=0}^{N}$ of $N+1$ vertices such that

$$
x_{0}=x, x_{N}=y \text { and }\left(x_{i-1}, x_{i}\right) \in E(G) \text { for } i=1, \ldots, N .
$$

A graph $G$ is connected if there is a path between any two vertices. $G$ is weakly connected if $\tilde{G}$ is connected.

If $G$ is such that $E(G)$ is symmetric and $x$ is a vertex in $G$, then the subgraph $G_{x}$ consisting of all edges and vertices which are contained in some path beginning at $x$ is called the component of $G$ containing $x$. In this case $V\left(G_{x}\right)=[x]_{G}$, where $[x]_{G}$ is the equivalence class of the following relation $R$ defined on $V(G)$ by the rule:

$y R z$ if there is a path in $G$ from $y$ to $z$.

Clearly, $G_{x}$ is connected.

\section{ItERATIONS AND FIXED POINTS OF BANACH $G$-CONTRACTIONS}

Throughout this section we assume that $(X, d)$ is a metric space, and $G$ is a directed graph such that $V(G)=X$ and $E(G) \supseteq \Delta$. The set of all fixed points of a mapping $f$ is denoted by Fix $f$.

Our first result shows that the convergence of successive aproximations for Banach $G$-contractions is closely related to the connectivity of a graph. We say that sequences $\left(x_{n}\right)_{n \in \mathbb{N}}$ and $\left(y_{n}\right)_{n \in \mathbb{N}}$, elements of $X$, are Cauchy equivalent if each of them is a Cauchy sequence and $d\left(x_{n}, y_{n}\right) \rightarrow 0$.

Theorem 3.1. The following statements are equivalent:

(i) $G$ is weakly connected;

(ii) for any $G$-contraction $f: X \rightarrow X$, given $x, y \in X$, the sequences $\left(f^{n} x\right)_{n \in \mathbb{N}}$ and $\left(f^{n} y\right)_{n \in \mathbb{N}}$ are Cauchy equivalent;

(iii) for any $G$-contraction $f: X \rightarrow X$, $\operatorname{card}($ Fix $f) \leq 1$.

We precede the proof of Theorem 3.1 with the following

Lemma 3.1. Let $f: X \rightarrow X$ be a $G$-contraction with a constant $\alpha$. Then, given $x \in X$ and $y \in[x]_{\tilde{G}}$, there is $r(x, y) \geq 0$ such that

$$
d\left(f^{n} x, f^{n} y\right) \leq \alpha^{n} r(x, y) \text { for all } n \in \mathbb{N} .
$$

Proof. Let $x \in X$ and $y \in[x]_{\tilde{G}}$. Then there is a path $\left(x_{i}\right)_{i=0}^{N}$ in $\tilde{G}$ from $x$ to $y$, i.e., $x_{0}=x, x_{N}=y$ and $\left(x_{i-1}, x_{i}\right) \in E(\tilde{G})$ for $i=1, \ldots, N$. By Proposition 2.1, $f$ is a $\tilde{G}$-contraction. An easy induction shows

$$
\left(f^{n} x_{i-1}, f^{n} x_{i}\right) \in E(\tilde{G}) \text { and } d\left(f^{n} x_{i-1}, f^{n} x_{i}\right) \leq \alpha^{n} d\left(x_{i-1}, x_{i}\right)
$$

for all $n \in \mathbb{N}$ and $i=1, \ldots, N$. Hence and by the triangle inequality, we get

$$
d\left(f^{n} x, f^{n} y\right) \leq \sum_{i=1}^{N} d\left(f^{n} x_{i-1}, f^{n} x_{i}\right) \leq \alpha^{n} \sum_{i=1}^{N} d\left(x_{i-1}, x_{i}\right),
$$

so it suffices to set $r(x, y):=\sum_{i=1}^{N} d\left(x_{i-1}, x_{i}\right)$. 
Proof of Theorem 3.1. (i) $\Rightarrow$ (ii): Let $f$ be a $G$-contraction and $x, y \in X$. By hypothesis, $[x]_{\tilde{G}}=X$, so $f x \in[x]_{\tilde{G}}$. By Lemma 3.1, we get

$$
d\left(f^{n} x, f^{n+1} x\right) \leq \alpha^{n} r(x, f x) \text { for all } n \in \mathbb{N} .
$$

Hence $\sum_{n=0}^{\infty} d\left(f^{n} x, f^{n+1} x\right)<\infty$ and a standard argument shows $\left(f^{n} x\right)_{n \in \mathbb{N}}$ is a Cauchy sequence. Since also $y \in[x]_{\tilde{G}}$, Lemma 3.1 yields $d\left(f^{n} x, f^{n} y\right) \leq \alpha^{n} r(x, y)$. Hence $\left(f^{n} x\right)_{n \in \mathbb{N}}$ and $\left(f^{n} y\right)_{n \in \mathbb{N}}$ are equivalent. Clearly, since $\left(f^{n} x\right)_{n \in \mathbb{N}}$ is a Cauchy sequence, so is $\left(f^{n} y\right)_{n \in \mathbb{N}}$.

(ii) $\Rightarrow$ (iii): Let $f$ be a $G$-contraction and $x, y \in$ Fix $f$. By (ii), $\left(f^{n} x\right)_{n \in \mathbb{N}}$ and $\left(f^{n} y\right)_{n \in \mathbb{N}}$ are equivalent which yields $x=y$.

(iii) $\Rightarrow(\mathrm{i})$ : Suppose, on the contrary, $G$ is not weakly connected, i.e., $\tilde{G}$ is disconnected. Let $x_{0} \in X$. Then both sets $\left[x_{0}\right]_{\tilde{G}}$ and $X \backslash\left[x_{0}\right]_{\tilde{G}}$ are nonempty. Let $y_{0} \in X \backslash\left[x_{0}\right]_{\tilde{G}}$ and define

$$
f x:=x_{0} \text { if } x \in\left[x_{0}\right]_{\tilde{G}} ; f x:=y_{0} \text { if } x \in X \backslash\left[x_{0}\right]_{\tilde{G}} .
$$

Clearly, Fix $f=\left\{x_{0}, y_{0}\right\}$. We show $f$ is a $G$-contraction. Let $(x, y) \in E(G)$. Then $[x]_{\tilde{G}}=[y]_{\tilde{G}}$, so either $x, y \in\left[x_{0}\right]_{\tilde{G}}$, or $x, y \in X \backslash\left[x_{0}\right]_{\tilde{G}}$. Hence in both cases $f x=f y$, so $(f x, f y) \in E(G)$ since $E(G) \supseteq \Delta$, and $d(f x, f y)=0 \leq 1 / 2 d(x, y)$. Thus $f$ is a $G$-contraction having two fixed points which violates (iii).

As an immediate consequence of Theorem 3.1, we obtain the following

Corollary 3.1. Let $(X, d)$ be complete. The following statements are equivalent:

(i) $G$ is weakly connected;

(ii) for any $G$-contraction $f: X \rightarrow X$, there is $x_{*} \in X$ such that $\lim _{n \rightarrow \infty} f^{n} x=$ $x_{*}$ for all $x \in X$.

The following example illuminates condition (i) of Corollary 3.1.

Example 3.1. Consider graphs $G_{1}$ and $G_{2}$ defined in Examples 2.3 and 2.4, respectively. Then $\tilde{G}_{1}=G_{2}=\tilde{G}_{2}$. The weak connectivity of $G_{1}$ or $G_{2}$ means given $x, y \in X$ there is a sequence $\left(x_{i}\right)_{i=0}^{N}$ such that $x_{0}=x, x_{N}=y$ and for all $i=1, \ldots, N$, $x_{i-1}$ and $x_{i}$ are comparable, i.e., $x_{i-1} \preceq x_{i}$ or $x_{i} \preceq x_{i-1}$. In particular, this is the case if we assume that (1.1) holds as done in fixed point theorems of Ran and Reurings [RR04], and Petruşel and Rus [PR06] or, more generally, (1.5) holds as done in corresponding theorems of Nieto and Rodríguez-López ([NR-L05], [NR-L07]; also [NPR-L07]). Indeed, both these conditions imply that for any $x, y \in X$, there is a path in $\tilde{G}_{1}$ (hence $\tilde{G}_{2}$ ) from $x$ to $y$ of length $\leq 2$. Clearly, even for $G_{1}$, (i) of Corollary 3.1 is more general than (1.1) or (1.5).

The next example shows that one cannot improve Corollary 3.1 by adding in (ii) that $x_{*}$ is a fixed point of $f$.

Example 3.2. Let $X:=[0,1]$ be endowed with the Euclidean metric $d_{E}$. Define the relation $\preceq$ in $X$ by

$$
x \preceq y \text { if } x, y \in(0,1] \text { and } x \leq y \text {, or } x=0 \text { and } y \in\{0,1\} .
$$

It is easily seen $\preceq$ is a partial order. (In fact, $(X, \preceq)$ is isomorphic to $\left(X^{\prime}, \preceq^{\prime}\right)$, where $X^{\prime}:=((0,1] \times\{0\}) \cup\{(1,-1)\}$ and $\preceq^{\prime}$ is the partial ordering with respect to the cone $[0, \infty)^{2}$.) Moreover, $(1.1)$ holds since 1 is the greatest element in $(X, \preceq)$. Hence and in view of Example 3.1, $G_{1}$ is weakly connected. Set

$$
f x:=x / 2 \text { for } x \in(0,1] \text {, and } f 0:=1 / 2 \text {. }
$$


It is easy to verify $f$ is $\preceq$-nondecreasing and (1.2) holds with $\alpha:=1 / 2$, i.e., $f$ is a $G_{1}$-contraction. Clearly, $f^{n} x \rightarrow 0$ for all $x \in X$, but $f$ has no fixed points.

The proofs of our fixed point theorems depend on the following

Proposition 3.1. Assume that $f: X \rightarrow X$ is a $G$-contraction such that for some $x_{0} \in X, f x_{0} \in\left[x_{0}\right]_{\tilde{G}}$. Let $\tilde{G}_{x_{0}}$ be the component of $\tilde{G}$ containing $x_{0}$. Then $\left[x_{0}\right]_{\tilde{G}}$ is $f$-invariant and $\left.f\right|_{\left[x_{0}\right]_{\tilde{G}}}$ is a $\tilde{G}_{x_{0}}$-contraction. Moreover, if $x, y \in\left[x_{0}\right]_{\tilde{G}}$, then $\left(f^{n} x\right)_{n \in \mathbb{N}}$ and $\left(f^{n} y\right)_{n \in \mathbb{N}}$ are Cauchy equivalent.

Proof. Let $x \in\left[x_{0}\right]_{\tilde{G}}$. Then there is a path $\left(x_{i}\right)_{i=0}^{N}$ in $\tilde{G}$ from $x_{0}$ to $x$, i.e., $x_{N}=x$ and $\left(x_{i-1}, x_{i}\right) \in E(\tilde{G})$ for $i=1, \ldots, N$. By Proposition 2.1, $f$ is a $\tilde{G}$-contraction which yields $\left(f x_{i-1}, f x_{i}\right) \in E(\tilde{G})$ for $i=1, \ldots, N$, i.e., $\left(f x_{i}\right)_{i=0}^{N}$ is a path in $\tilde{G}$ from $f x_{0}$ to $f x$. Thus $f x \in\left[f x_{0}\right]_{\tilde{G}}$. Since, by hypothesis, $f x_{0} \in\left[x_{0}\right]_{\tilde{G}}$, i.e., $\left[f x_{0}\right]_{\tilde{G}}=\left[x_{0}\right]_{\tilde{G}}$, we infer $f x \in\left[x_{0}\right]_{\tilde{G}}$. Thus $\left[x_{0}\right]_{\tilde{G}}$ is $f$-invariant.

Now let $(x, y) \in E\left(\tilde{G}_{x_{0}}\right)$. This means there is a path $\left(x_{i}\right)_{i=0}^{N}$ in $\tilde{G}$ from $x_{0}$ to $y$ such that $x_{N-1}=x$. Let $\left(y_{i}\right)_{i=0}^{M}$ be a path in $\tilde{G}$ from $x_{0}$ to $f x_{0}$. Repeating the argument from the first part of the proof, we infer $\left(y_{0}, y_{1}, \ldots, y_{M}, f x_{1}, \ldots, f x_{N}\right)$ is a path in $\tilde{G}$ from $x_{0}$ to $f y$; in particular, $\left(f x_{N-1}, f x_{N}\right) \in E\left(\tilde{G}_{x_{0}}\right)$, i.e., $(f x, f y) \in$ $E\left(\tilde{G}_{x_{0}}\right)$. Moreover, since $E\left(\tilde{G}_{x_{0}}\right) \subseteq E(\tilde{G})$ and $f$ is a $\tilde{G}$-contraction, we infer (2.3) holds for the graph $\tilde{G}_{x_{0}}$. Thus $\left.f\right|_{\left[x_{0}\right]_{\tilde{G}}}$ is a $\tilde{G}_{x_{0}}$-contraction.

Finally, in view of Theorem 3.1, the second statement follows immediately from the first one since $\tilde{G}_{x_{0}}$ is connected.

Theorem 3.2. Let $(X, d)$ be complete, and let the triple $(X, d, G)$ have the following property:

for any $\left(x_{n}\right)_{n \in \mathbb{N}}$ in $X$, if $x_{n} \rightarrow x$ and $\left(x_{n}, x_{n+1}\right) \in E(G)$ for $n \in \mathbb{N}$, then there is a subsequence $\left(x_{k_{n}}\right)_{n \in \mathbb{N}}$ with $\left(x_{k_{n}}, x\right) \in E(G)$ for $n \in \mathbb{N}$.

Let $f: X \rightarrow X$ be a $G$-contraction, and $X_{f}:=\{x \in X:(x, f x) \in E(G)\}$. Then the following statements hold.

$1^{o}$ card Fix $f=\operatorname{card}\left\{[x]_{\tilde{G}}: x \in X_{f}\right\}$.

$2^{o}$ Fix $f \neq \emptyset$ iff $X_{f} \neq \emptyset$.

$3^{o} f$ has a unique fixed point iff there exists $x_{0} \in X_{f}$ such that $X_{f} \subseteq\left[x_{0}\right]_{\tilde{G}}$.

$4^{o}$ For any $x \in X_{f},\left.f\right|_{[x]_{\tilde{G}}}$ is a $P O$.

$5^{o}$ If $X_{f} \neq \emptyset$ and $G$ is weakly connected, then $f$ is a $P O$.

$6^{o}$ If $X^{\prime}:=\bigcup\left\{[x]_{\tilde{G}}: x \in X_{f}\right\}$, then $\left.f\right|_{X^{\prime}}$ is a WPO.

$7^{\circ}$ If $f \subseteq E(G)$, then $f$ is a WPO.

Proof. We begin with points $4^{o}$ and $5^{\circ}$. Let $x \in X_{f}$. Then $f x \in[x]_{\tilde{G}}$, so by Proposition 3.1, if $y \in[x]_{\tilde{G}}$, then $\left(f^{n} x\right)_{n \in \mathbb{N}}$ and $\left(f^{n} y\right)_{n \in \mathbb{N}}$ are Cauchy equivalent. By completeness, $\left(f^{n} x\right)_{n \in \mathbb{N}}$ converges to some $x_{*} \in X$. Clearly, also $\lim _{n \rightarrow \infty} f^{n} y=x_{*}$. Since $(x, f x) \in E(G),(2.2)$ yields

$$
\left(f^{n} x, f^{n+1} x\right) \in E(G) \text { for } n \in \mathbb{N} .
$$

By (3.1), there is a subsequence $\left(f^{k_{n}} x\right)_{n \in \mathbb{N}}$ such that $\left(f^{k_{n}} x, x_{*}\right) \in E(G)$ for $n \in \mathbb{N}$. Hence and by (3.2), we infer $\left(x, f x, f^{2} x, \ldots, f^{k_{1}} x, x_{*}\right)$ is a path in $G$ (hence also in $\tilde{G})$ from $x$ to $x_{*}$, i.e., $x_{*} \in[x]_{\tilde{G}}$. Moreover, by $(2.3)$,

$$
d\left(f^{k_{n}+1} x, f x_{*}\right) \leq \alpha d\left(f^{k_{n}} x, x_{*}\right) \text { for } n \in \mathbb{N} .
$$


Hence, letting $n$ tend to $\infty$ we conclude $x_{*}=f x_{*}$. Thus $\left.f\right|_{[x]_{\tilde{G}}}$ is a PO. Moreover, if $G$ is weakly connected, then $[x]_{\tilde{G}}=X$, so $f$ is a PO.

Now $6^{\circ}$ is an easy consequence of $4^{\circ}$. To show $7^{\circ}$ observe that $f \subseteq E(G)$ means $X_{f}=X$. This yields $X^{\prime}=X$, so $f$ is a WPO in view of $6^{\circ}$.

To prove $1^{o}$, consider a mapping $\pi$ defined by

$$
\pi(x):=[x]_{\tilde{G}} \text { for all } x \in \operatorname{Fix} f .
$$

It suffices to show $\pi$ is a bijection of Fix $f$ onto $\mathcal{C}:=\left\{[x]_{\tilde{G}}: x \in X_{f}\right\}$. Since $E(G) \supseteq$ $\Delta$, we infer Fix $f \subseteq X_{f}$ which yields $\pi($ Fix $f) \subseteq \mathcal{C}$. On the other hand, if $x \in X_{f}$, then by $4^{o}, \lim _{n \rightarrow \infty} f^{n} x \in[x]_{\tilde{G}} \cap$ Fix $f$ which implies $\pi\left(\lim _{n \rightarrow \infty} f^{n} x\right)=[x]_{\tilde{G}}$. Thus $f$ is a surjection of Fix $f$ onto $\mathcal{C}$. Now, if $x_{1}, x_{2} \in$ Fix $f$ are such that $\pi\left(x_{1}\right)=\pi\left(x_{2}\right)$, i.e., $\left[x_{1}\right]_{\tilde{G}}=\left[x_{2}\right]_{\tilde{G}}$, then $x_{2} \in\left[x_{1}\right]_{\tilde{G}}$, so by $4^{o}$,

$$
\lim _{n \rightarrow \infty} f^{n} x_{2} \in\left[x_{1}\right]_{\tilde{G}} \cap \operatorname{Fix} f=\left\{x_{1}\right\},
$$

i.e., $x_{2}=x_{1}$ since $f^{n} x_{2}=x_{1}$. Consequently, $f$ is injective. Thus $1^{o}$ is proved.

Finally, observe that $2^{\circ}$ and $3^{\circ}$ are simple consequences of $1^{\circ}$.

Remark 3.1. For a triple $\left(X, d, G_{2}\right)$, property (3.1) was introduced by Nieto and Rodríguez-López [NR-L07]. If we assume that a graph $G$ is such that $E(G)$ is a quasi-order (i.e., it is transitive), then (3.1) is equivalent to the following:

$$
\begin{gathered}
\text { for any }\left(x_{n}\right)_{n \in \mathbb{N}} \text {, if } x_{n} \rightarrow x \text { and }\left(x_{n}, x_{n+1}\right) \in E(G) \text { for } n \in \mathbb{N}, \\
\text { then }\left(x_{n}, x\right) \in E(G) \text { for } n \in \mathbb{N} \text {. }
\end{gathered}
$$

Indeed, (3.3) $\Rightarrow(3.1)$ is trivial. Now assume (3.1) holds and $\left(x_{n}\right)_{n \in \mathbb{N}}$ is as in (3.3). By transitivity, $\left(x_{n}, x_{m}\right) \in E(G)$ if $n \leq m$. By $(3.1)$, for some $\left(k_{n}\right)_{n \in \mathbb{N}},\left(x_{k_{n}}, x\right) \in E(G)$ for $n \in \mathbb{N}$. Since $n \leq k_{n}$, we have $\left(x_{n}, x_{k_{n}}\right) \in E(G)$. By transitivity, we infer $\left(x_{n}, x\right) \in E(G)$.

On the other hand, if a triple $(X, d, G)$ has property $(3.3)$, then $E(G)$ is a quasiorder. Indeed, if $(x, y) \in E(G)$ and $(y, z) \in E(G)$, then applying $(3.3)$ to $\left(x_{n}\right)_{n \in \mathbb{N}}$ defined by

$$
x_{1}:=x, x_{2}:=y, x_{n}:=z \text { for } n \geq 3,
$$

we get $\left(x_{n}, z\right) \in E(G)$ for $n \in \mathbb{N}$, and hence $(x, z) \in E(G)$.

For a triple $\left(X, d, G_{1}\right)$, property (3.3) was introduced by Nieto and RodríguezLópez [NR-L05].

Remark 3.2. Obviously, the graph $G_{0}$ (cf. Example 2.2) is connected, so Theorem 3.2 yields the Banach Principle. On the other hand, it is not clear whether Theorems 1.1 and 1.2 generalize Banach's theorem. With the help of Zermelo's wellordering theorem, we may observe only that the Banach Principle can be derived from Theorem 1.3.

Remark 3.3. Condition $f \subseteq E\left(G_{1}\right)$ (cf. Example 2.3) of point $7^{\circ}$ of Theorem 3.2 means $f$ is progressive, i.e., $x \preceq f x$ for all $x \in X$. By Zermelo's fixed point theorem (see, e.g., [DS58, p. 5]), each progressive selfmap of a partially ordered set has a fixed point if every chain has a supremum. (For a discussion on applications of Zermelo's theorem in metric fixed point theory, see [Ja01].) However, in many cases the last condition turns out to be too strong; in particular, it does not hold for $(\mathbb{R}, \leq)$. On the other hand, for the graph $G_{1},(3.3)$ is satisfied if $\left(X, d, G_{1}\right)$ has the following property: every countable relatively compact chain $C$ has a supremum, and $\sup C \in \mathrm{cl} C$. (To prove it, use a similar argument as in the proof of [NR-L05, Lemma 1].) Clearly, the last property holds for the Euclidean space $\mathbb{R}$. 
Corollary 3.2. Let $(X, d)$ be complete, and let the triple $(X, d, G)$ have property (3.1). The following statements are equivalent:

(i) $G$ is weakly connected;

(ii) every $G$-contraction $f: X \rightarrow X$ such that $\left(x_{0}, f x_{0}\right) \in E(G)$ for some $x_{0} \in$ $X$, is a $P O$;

(iii) for any $G$-contraction $f: X \rightarrow X$, card Fix $f \leq 1$.

Proof. (i) $\Rightarrow$ (ii) follows directly from Theorem $3.2\left(5^{\circ}\right)$.

(ii) $\Rightarrow$ (iii): Let $f: X \rightarrow X$ be a $G$-contraction. If $X_{f}$ is empty, so is Fix $f$ since Fix $f \subseteq X_{f}$. If $X_{f} \neq \emptyset$, then by (ii), Fix $f$ is a singleton. In both cases card Fix $f \leq 1$.

(iii) $\Rightarrow$ (i) follows from Theorem 3.1.

Remark 3.4. Corollary 3.2 illuminates [NR-L05, Ex. 1] where $G_{1}$-contractions on subsets of $\mathbb{R}^{2}$ were defined having more than one fixed point. By Corollary 3.2, such examples exist for any triple $\left(X, d, G_{1}\right)$ such that $G_{1}$ is not weakly connected.

Our next result gives another sufficient condition for the existence of fixed points in the case where a triple $(X, d, G)$ may fail to have property (3.1).

Theorem 3.3. Let $(X, d)$ be complete, and let $f: X \rightarrow X$ be a $G$-contraction such that $f$ is orbitally $G$-continuous. Let $X_{f}:=\{x \in X:(x, f x) \in E(G)\}$. Then the following statements hold.

$1^{o}$ Fix $f \neq \emptyset$ iff $X_{f} \neq \emptyset$.

$2^{o}$ For any $x \in X_{f}$ and $y \in[x]_{\tilde{G}}$, $\left(f^{n} y\right)_{n \in \mathbb{N}}$ converges to a fixed point of $f$ and $\lim _{n \rightarrow \infty} f^{n} y$ does not depend on $y$.

$3^{o}$ If $X_{f} \neq \emptyset$ and $G$ is weakly connected, then $f$ is a PO.

$4^{o}$ If $f \subseteq E(G)$, then $f$ is a WPO.

Proof. We begin with point $2^{\circ}$. Let $x \in X$ be such that $(x, f x) \in E(G)$, and let $y \in[x]_{\tilde{G}}$. By Proposition 3.1, $\left(f^{n} x\right)_{n \in \mathbb{N}}$ and $\left(f^{n} y\right)_{n \in \mathbb{N}}$ converge to the same point $x_{*}$. Moreover, $\left(f^{n} x, f^{n+1} x\right) \in E(G)$ for $n \in \mathbb{N}$. Since $f$ is orbitally $G$ continuous, we get $f\left(f^{n} x\right) \rightarrow f x_{*}$. This yields $x_{*}=f x_{*}$ since, simultaneously, $f\left(f^{n} x\right)=f^{n+1} x \rightarrow x_{*}$. Thus we proved $2^{o}$ and ' $\Leftarrow$ ' of $1^{o}$. ' $\Rightarrow$ ' of $1^{o}$ follows from the assumption $E(G) \supseteq \Delta$. $4^{\circ}$ is an immediate consequence of $2^{\circ}$ since $f \subseteq E(G)$ means $X_{f}=X$. To prove $3^{o}$ observe that if $x_{0} \in X_{f}$, then $\left[x_{0}\right]_{\tilde{G}}=X$, so $2^{o}$ yields $f$ is a $\mathrm{PO}$.

Now we give another version of Theorem 3.3 in which we slightly strengthen a continuity condition on $f$.

Theorem 3.4. Let $(X, d)$ be complete, and let $f: X \rightarrow X$ be a $G$-contraction such that $f$ is orbitally continuous. Then the following statements hold.

$1^{o}$ Fix $f \neq \emptyset$ iff there exists $x_{0} \in X$ with $f x_{0} \in\left[x_{0}\right]_{\tilde{G}}$.

$2^{o}$ If $x \in X$ is such that $f x \in[x]_{\tilde{G}}$, then for any $y \in[x]_{\tilde{G}},\left(f^{n} y\right)_{n \in \mathbb{N}}$ converges to a fixed point of $f$, and $\lim _{n \rightarrow \infty} f^{n} y$ does not depend on $y$.

$3^{\circ}$ If $G$ is weakly connected, then $f$ is a PO.

$4^{o}$ If $f x \in[x]_{\tilde{G}}$ for any $x \in X$, then $f$ is a WPO.

Proof. We begin with $2^{o}$. Let $x \in X$ be such that $f x \in[x]_{\tilde{G}}$, and let $y \in[x]_{\tilde{G}}$. By Proposition 3.1, $\left(f^{n} x\right)_{n \in \mathbb{N}}$ and $\left(f^{n} y\right)_{n \in \mathbb{N}}$ converge to the same point $x_{*}$. Since $f$ is orbitally continuous, $f\left(f^{n} x\right) \rightarrow f x_{*}$ which yields $x_{*}=f x_{*}$. So $2^{o}$ and ' $\Leftarrow$ ' of $1^{o}$ 
are proved. ' $\Rightarrow$ ' of $1^{o}$ is obvious since $x \in[x]_{\tilde{G}}$ for any $x \in X$. Now if $G$ is weakly connected, then for any $x \in X,[x]_{\tilde{G}}=X$; in particular, $f x \in[x]_{\tilde{G}}$, and by $2^{o}$, we infer $f$ is a PO. Thus $3^{\circ}$ holds. Finally, $4^{o}$ is an immediate consequence of $2^{\circ}$.

Remark 3.5. In view of Proposition 3.1, condition ' $f x \in[x]_{\tilde{G}}$ for any $x \in X$ ' used in $4^{o}$ of Theorem 3.4 means all components of $\tilde{G}$ are $f$-invariant whereas $1^{o}$ is equivalent to the statement: $f$ has a fixed point iff some component of $\tilde{G}$ is $f$-invariant.

Remark 3.6. We emphasize that the advantage of Theorem $3.4\left(3^{\circ}\right)$ over Theorems $3.2\left(5^{\circ}\right)$ and $3.3\left(3^{\circ}\right)$ is that we need not verify here if there is $x_{0} \in X$ with $\left(x_{0}, f x_{0}\right) \in E(G)$. In particular, Theorem 3.4 extends essentially results of Ran and Reurings [RR04, Th. 2.1], Petruşel and Rus [PR06, Th. 4.3], and Nieto and Rodríguez-López [NR-L05, Ths. 2.1 and 2.3].

Corollary 3.3. Let $(X, d)$ be complete. The following statements are equivalent:

(i) $G$ is weakly connected;

(ii) every orbitally continuous $G$-contraction is a $P O$;

(iii) for any orbitally continuous $G$-contraction $f: X \rightarrow X$, card Fix $f \leq 1$.

Hence if $\tilde{G}$ is disconnected, then there exists an orbitally continuous G-contraction which has at least two fixed points.

Proof. Theorem $3.4\left(3^{\circ}\right)$ yields (i) $\Rightarrow($ ii). (ii) $\Rightarrow($ iii) is obvious. (iii) $\Rightarrow$ (i) follows from the proof of (iii) $\Rightarrow$ (i) of Theorem 3.1; observe that $f$ defined there is orbitally continuous.

Remark 3.7. The assumptions of Theorems 3.3 and 3.4 seem to be very close: the only difference concerns the type of continuity of $f$. Thus the question arises whether we could improve Theorem $3.3\left(1^{\circ}\right)$ by substituting condition ' $f x_{0} \in\left[x_{0}\right]_{\tilde{G}}$ ' for ' $\left(x_{0}, f x_{0}\right) \in E(G)$ '. However, the answer is negative. To see that, consider again Example 3.2. Here $f$ is not orbitally continuous since $f^{n} 1 \rightarrow 0$, but $f\left(f^{n} 1\right) \nrightarrow f 0$. On the other hand, $f$ is $G_{1}$-continuous (hence orbitally $G_{1}$-continuous). Indeed, let $\left(x_{n}\right)_{n \in \mathbb{N}}$ be such that $x_{n} \preceq x_{n+1}$ for $n \in \mathbb{N}$, and $x_{n} \rightarrow x$. If $x_{n} \in(0,1]$ for $n \in \mathbb{N}$, then $x_{n} \nearrow x$, so $f x_{n} \rightarrow f x$ since $f$ is $d_{E}$-continuous from the left. If $x_{k}=0$ for some $k \in \mathbb{N}$, then either $\left(x_{n}\right)_{n \in \mathbb{N}}=(0,0, \ldots)$, or $\left(x_{n}\right)_{n \in \mathbb{N}}=(0, \ldots, 1,1, \ldots)$. In both cases $f x_{n} \rightarrow f x$. Moreover, observe that in each case $x_{n} \preceq x$. So the above argument also shows $(X, d, \preceq)$ has property (1.3). Since $f$ has no fixed points and $G_{1}$ is weakly connected, we infer that we can improve neither Theorem $3.3\left(1^{\circ}\right.$, $\left.3^{\circ}\right)$ nor Theorem $3.2\left(2^{\circ}, 5^{\circ}\right)$ by modifying or removing condition ' $X_{f} \neq \emptyset$ ', as done in Theorem $3.4\left(1^{\circ}, 3^{\circ}\right)$. In particular, the assumption 'there is $x_{0} \in X$ with $x_{0} \preceq f x_{0}$ or $f x_{0} \preceq x_{0}$ ' is unnecessary in Theorem 1.1 , but it cannot be dropped in Theorem 1.2 unless $f$ is orbitally continuous.

The following example shows that under the assumptions of Theorem 3.4, given $x \in X_{f}$, neither $\left.f\right|_{[x]_{\tilde{G}}}$ nor $\left.f\right|_{\mathrm{cl}[x]_{\tilde{G}}}$ need be a PO. So we cannot improve Theorem 3.4 by formulating statement $2^{\circ}$ in a similar way as $4^{\circ}$ of Theorem 3.2.

Example 3.3. Let $X:=[0,1]$ be endowed with the Euclidean metric. Define the relation $\preceq$ in $X$ by

$$
x \preceq y \text { if } x, y \in(0,1) \text { and } x \leq y \text {, or } x=0 \text { and } y=0 \text {, or } x=1 \text { and } y=1 .
$$


Then $\preceq$ is a partial order. Set

$$
f x:=x / 2 \text { for } x \in[0,1) \text { and } f 1:=1 .
$$

Let $G_{1}$ be the graph defined in Example 2.3. It is easily seen that $f$ is a $G_{1^{-}}$ contraction and $f$ is orbitally continuous, so the assumptions of Theorem 3.3 are satisfied. However, for $x \in(0,1),[x]_{\tilde{G}}=(0,1)$, and hence $\left.f\right|_{[x]_{\tilde{G}}}$ has no fixed points, while $\left.f\right|_{\operatorname{cl}[x]_{\tilde{G}}}(=f)$ has two fixed points.

Nevertheless, some improvements of Theorem 3.4 in the above-mentioned direction are possible under some stronger assumptions on $f$ as done in the following

Theorem 3.5. Let $(X, d)$ be complete, and let $f: X \rightarrow X$ be a $G$-contraction such that $f$ is nonexpansive or the family $\left\{f^{n}: n \in \mathbb{N}\right\}$ is equicontinuous on $X$. Then, for any $x \in X$ such that $f x \in[x]_{\tilde{G}},\left.f\right|_{\operatorname{cl}[x]_{\tilde{G}}}$ is a PO.

We precede the proof of Theorem 3.5 by the following result. (Incidentally, it also implies the so-called double contraction principle of Lasota [L95].)

Lemma 3.2. Let $E$ be a dense subset of $X$, and let $f: X \rightarrow X$ be such that for some $x_{*} \in X$,

$$
f^{n} x \rightarrow x_{*} \text { for } x \in E .
$$

If the family $\left\{f^{n}: n \in \mathbb{N}\right\}$ is equicontinuous on $X$, then $f^{n} x \rightarrow x_{*}$ for all $x \in X$.

Proof. Let $x \in X$ and $\varepsilon>0$. Since $\left\{f^{n}: n \in \mathbb{N}\right\}$ is equicontinuous at $x$, there is $\delta>0$ such that if $y \in X$ and $d(x, y)<\delta$, then $d\left(f^{n} x, f^{n} y\right)<\varepsilon / 2$ for $n \in \mathbb{N}$. Since $\operatorname{cl} E=X$, there is $y \in E$ such that $d(x, y)<\delta$. By hypothesis, $f^{n} y \rightarrow x_{*}$ so there is $k \in \mathbb{N}$ such that $d\left(f^{n} y, x_{*}\right)<\varepsilon / 2$ for $n \geq k$. Hence we get

$$
d\left(f^{n} x, x_{*}\right) \leq d\left(f^{n} x, f^{n} y\right)+d\left(f^{n} y, x_{*}\right)<\varepsilon \text { for } n \geq k
$$

which yields $f^{n} x \rightarrow x_{*}$.

Proof of Theorem 3.5. Since $\left\{f^{n}: n \in \mathbb{N}\right\}$ is equicontinuous if $f$ is nonexpansive, it suffices to consider the case where the former condition holds. Assume $f x \in[x]_{\tilde{G}}$. By Proposition 3.1, $[x]_{\tilde{G}}$ is $f$-invariant. Hence and by continuity of $f, \operatorname{cl}[x]_{\tilde{G}}$ is $f$-invariant. By Theorem 3.4, there is $x_{*} \in \operatorname{Fix} f$ such that $f^{n} y \rightarrow x_{*}$ for $y \in[x]_{\tilde{G}}$. Clearly, $x_{*} \in \operatorname{cl}[x]_{\tilde{G}}$. Since $\left\{f^{n}: n \in \mathbb{N}\right\}$ is equicontinuous, we infer in view of Lemma 3.2 that $f^{n} y \rightarrow x_{*}$ for $y \in \operatorname{cl}[x]_{\tilde{G}}$ which completes the proof.

Now we wish to present that even for particular graphs, Theorems 3.2-3.5 extend and subsume a number of recent results of other authors.

Remark 3.8. Let $G:=G_{1}$ as in Example 2.3. Then Theorems $3.2\left(2^{\circ}, 5^{\circ}\right)$ and $3.3\left(1^{\circ}, 3^{\circ}\right)$ generalize the results of Nieto and Rodríguez-López [NR-L05, Ths. $2.1-2.3]$.

Remark 3.9. Let $G:=G_{1}^{-1}$. Observe that a mapping $f$ is $\preceq$-nondecreasing iff $f$ is $\succeq$-nondecreasing; moreover, a sequence $\left(x_{n}\right)_{n \in \mathbb{N}}$ is $\preceq$-nonincreasing iff it is $\succeq$ nondecreasing. Hence, for that graph, Theorems $3.2\left(2^{\circ}, 5^{\circ}\right)$ and $3.3\left(1^{\circ}, 3^{\circ}\right)$ yield [NR-L05, Ths. 2.4-2.5].

Remark 3.10. For $G:=G_{2}$ as in Example 2.4, Theorem $3.3\left(2^{\circ}, 5^{\circ}\right)$ extends the results of Ran and Reurings [RR04, Th. 2.1], and Petruşel and Rus [PR06, Ths. 1.2 and 4.3] (here the so-called compatibility conditions between a metric and an order structure can be dropped). Also, for the same graph, Theorems $3.2\left(5^{\circ}\right)$ and 3.3 
$\left(3^{\circ}\right)$ improve results of Nieto and Rodríguez-López [NR-L07, Ths. 4, 5 and 7] and [NPR-L07, Th. 3.1]. Indeed, we weakened the authors' hypothesis (1.5) by assuming the weak connectivity of graph $G$ (cf. Example 3.1), and we also relaxed their continuity assumption on a mapping.

On the other hand, Theorem 3.4 yields directly the following well-known fixed point theorem which is quite different from the above results.

Corollary 3.4 (Edelstein). Let $(X, d)$ be complete and $\varepsilon$-chainable for some $\varepsilon>0$, i.e., given $x, y \in X$, there is $N \in \mathbb{N}$ and a sequence $\left(x_{i}\right)_{i=0}^{N}$ such that

$$
x_{0}=x, x_{N}=y \text { and } d\left(x_{i-1}, x_{i}\right)<\varepsilon \text { for } i=1, \ldots, N \text {. }
$$

Let $f: X \rightarrow X$ be such that

$$
\exists_{\alpha \in(0,1)} \forall_{x, y \in X}(d(x, y)<\varepsilon \Rightarrow d(f x, f y) \leq \alpha d(x, y)) .
$$

Then $f$ is a $P O$.

Proof. Clearly, (3.4) implies $f$ is continuous. Consider the graph $G$ with $V(G):=$ $X$, and

$$
E(G):=\{(x, y) \in X \times X: d(x, y)<\varepsilon\} .
$$

Then $\varepsilon$-chainability of $(X, d)$ means $G$ is connected. If $(x, y) \in E(G)$, then $(3.4)$ yields

$$
d(f x, f y) \leq \alpha d(x, y)<\alpha \varepsilon<\varepsilon .
$$

Hence (2.2) and (2.3) hold, so $f$ is a $G$-contraction. By Theorem $3.4\left(3^{\circ}\right), f$ is a PO.

Remark 3.11. Let $G$ be defined as in the proof of Corollary 3.4. Then $(X, d, G)$ has property (3.1). Indeed, if $x_{n} \rightarrow x$, then $d\left(x_{n}, x\right)<\varepsilon$ for sufficiently large $n$, so there is $\left(x_{k_{n}}\right)_{n \in \mathbb{N}}$ such that $\left(x_{k_{n}}, x\right) \in E(G)$. Thus, also Theorem 3.2 is applicable here. In particular, if $(X, d)$ is not $\varepsilon$-chainable, then by Theorem $3.2\left(1^{\circ}\right)$, we get a characterization of card Fix $f$ for an Edelstein contraction $f$. In this case we may also apply Theorem 3.5 since (3.4) implies $\left\{f^{n}: n \in \mathbb{N}\right\}$ is equicontinuous on $X$.

The following result will be useful in the next section.

Proposition 3.2. If $E(G)$ is a quasi-order and given $x \in X$, the set $\{y \in X$ : $(x, y) \in E(G)\}$ is closed, then $(X, d, G)$ has property (3.3).

Proof. Let $\left(x_{n}\right)_{n \in \mathbb{N}}$ be such that $\left(x_{n}, x_{n+1}\right) \in E(G)$ for $n \in \mathbb{N}$ and $x_{n} \rightarrow x$. By transitivity, given $n \in \mathbb{N}$,

$$
x_{m} \in\left\{y \in X:\left(x_{n}, y\right) \in E(G)\right\} \text { for } m \geq n .
$$

Letting $m$ tend to $\infty$, in view of the hypothesis we get $\left(x_{n}, x\right) \in E(G)$.

\section{Application: A generalization of the Kelisky-Rivlin theorem}

Recall that the Bernstein operator $B_{n}(n \in \mathbb{N})$ on the space $C[0,1]$ of all continuous real functions on the closed unit interval is defined by

$$
\left(B_{n} \varphi\right)(t):=\sum_{k=0}^{n} \varphi\left(\frac{k}{n}\right)\left(\begin{array}{l}
n \\
k
\end{array}\right) t^{k}(1-t)^{n-k} \text { for } \varphi \in C[0,1] \text { and } t \in[0,1] \text {. }
$$


Kelisky and Rivlin [KR67] proved that each Bernstein operator $B_{n}$ is a WPO. Moreover, given $n \in \mathbb{N}$ and $\varphi \in C[0,1]$,

$$
\lim _{j \rightarrow \infty}\left(B_{n}^{j} \varphi\right)(t)=\varphi(0)+(\varphi(1)-\varphi(0)) t \text { for } t \in[0,1] .
$$

In their opinion the study of iterates of $B_{n}$ is considerably simplified if one uses the language of linear algebra. Nevertheless, their proof is not easy: in particular, it involves the Stirling numbers of the second kind, and eigenvalues and eigenvectors of some matrices. Recently, a simple proof of the Kelisky-Rivlin theorem was given by Rus [R04] with the help of some trick with the Contraction Principle. Our purpose here is to show that the Bernstein operator $B_{n}$ is a Banach $G$-contraction for some graph $G$ such that $B_{n} \subseteq E(G)$, and hence, in view of Theorem 3.3, $B_{n}$ is a WPO. In fact, this is a consequence of the following more general result which extends the Kelisky-Rivlin theorem.

Theorem 4.1. Let $X$ be a Banach space and $X_{0}$ a closed subspace of $X$. Let $T: X \rightarrow X$ be a linear operator (not necessarily continuous on $X$ ) such that $\left\|\left.T\right|_{X_{0}}\right\|<1$. If the corresponding field $I-T$ is such that $(I-T)(X) \subseteq X_{0}$, then $T$ is a WPO. Moreover, $\operatorname{card}$ Fix $T=\operatorname{card} X / X_{0}$, and

$$
\left(x+X_{0}\right) \cap \operatorname{Fix} T=\left\{\lim _{n \rightarrow \infty} T^{n} x\right\} \text { for } x \in X .
$$

Proof. Define the following graph $G: V(G):=X$ and for $x, y \in X$,

$$
(x, y) \in E(G) \text { if } x-y \in X_{0} .
$$

Clearly, $E(G)$ is an equivalence relation; in particular, $E(G) \supseteq \Delta$ and by symmetry, $\tilde{G}=G$. We show both Theorems 3.2 and 3.3 are applicable here. First we prove $T$ is a $G$-contraction. Let $x, y \in E(G)$, i.e., $x-y \in X_{0}$. Then we have

$$
T x-T y=(y-T y)-(x-T x)+(x-y) \in X_{0},
$$

since, by hypothesis, $y-T y, x-T x \in X_{0}$. Thus $(T x, T y) \in E(G)$ and, moreover,

$$
\|T x-T y\|=\|T(x-y)\| \leq\left\|\left.T\right|_{X_{0}}\right\|\|x-y\| .
$$

Since $\left\|\left.T\right|_{X_{0}}\right\|<1$, we infer $T$ is a $G$-contraction.

Observe that given $x \in X$,

$$
\{y \in X:(x, y) \in E(G)\}=x+X_{0} .
$$

Since $X_{0}$ is closed, so is $x+X_{0}$. Thus Proposition 3.2 implies $(X, d, G)$ has property (3.3) since, in particular, $E(G)$ is a quasi-order.

We show $T$ is $G$-continuous. Let $\left(x_{n}, x_{n+1}\right) \in E(G)$ for $n \in \mathbb{N}$ and $x_{n} \rightarrow x$. By transitivity, $\left(x_{1}, x_{n}\right) \in E(G)$, i.e., $x_{1}-x_{n} \in X_{0}$. Since $X_{0}$ is closed, we infer $x_{1}-x \in X_{0}$. Hence and by continuity of $\left.T\right|_{X_{0}}$, we get $T\left(x_{1}-x_{n}\right) \rightarrow T\left(x_{1}-x\right)$. This yields $T x_{n} \rightarrow T x$ since $T$ is linear. Consequently, $T$ is $G$-continuous.

Now condition $(I-T)(X) \subseteq X_{0}$ means $(x, T x) \in E(G)$ for $x \in X$, i.e., $T \subseteq E(G)$. So both Theorems 3.2 and 3.3 imply $T$ is a WPO. Moreover, since $E(\tilde{G})(=E(G))$ is transitive, we infer that given $x \in X$,

$$
[x]_{\tilde{G}}=\{y \in X:(x, y) \in E(G)\}=x+X_{0} .
$$

Hence and by Theorem $3.2\left(1^{\circ}\right)$,

$$
\operatorname{card} \text { Fix } T=\operatorname{card}\left\{x+X_{0}: x \in X_{T}\right\}=\operatorname{card} X / X_{0}
$$

since $X_{T}=X$. Finally, Theorem $3.2\left(4^{\circ}\right)$ yields the last statement of the thesis. 
Now we show the Kelisky-Rivlin theorem is a particular case of Theorem 4.1. Set $X:=C[0,1]$ and

$$
X_{0}:=\{\varphi \in X: \varphi(0)=\varphi(1)=0\} .
$$

We endow $X$ with the sup norm. Observe that $\left(B_{1} \varphi\right)(t)=\varphi(0)(1-t)+\varphi(1) t$. Hence $\left.B_{1}\right|_{X_{0}}=0$, and

$$
\left(I-B_{1}\right)(\varphi)(t)=\varphi(t)-\varphi(0)(1-t)-\varphi(1) t
$$

which yields $\left(I-B_{1}\right)(X) \subseteq X_{0}$.

Now let $n \in \mathbb{N}$ and $n \geq 2$. Given $\varphi \in X_{0}$ and $t \in[0,1]$, we have

$$
\begin{aligned}
\left|\left(B_{n} \varphi\right)(t)\right| & =\left|\sum_{k=1}^{n-1} \varphi\left(\frac{k}{n}\right)\left(\begin{array}{l}
n \\
k
\end{array}\right) t^{k}(1-t)^{n-k}\right| \leq \sum_{k=1}^{n-1}\left(\begin{array}{l}
n \\
k
\end{array}\right) t^{k}(1-t)^{n-k}\|\varphi\| \\
& =\left(1-t^{n}-(1-t)^{n}\right)\|\varphi\| \leq\left(1-1 / 2^{n-1}\right)\|\varphi\|
\end{aligned}
$$

which yields $\left.|| B_{n}\right|_{X_{0}}||<1$. We show $\left(I-B_{n}\right)(X) \subseteq X_{0}$. Let $\varphi \in X$ and $t \in[0,1]$. Then we have

$$
\left(I-B_{n}\right)(\varphi)(t)=\sum_{k=0}^{n}\left(\varphi(t)-\varphi\left(\frac{k}{n}\right)\right)\left(\begin{array}{l}
n \\
k
\end{array}\right) t^{k}(1-t)^{n-k} .
$$

Hence it is clear that $\left(I-B_{n}\right)(\varphi) \in X_{0}$. By Theorem 4.1, $B_{n}$ is a WPO and given $\varphi \in X$,

$$
\left(\varphi+X_{0}\right) \cap \operatorname{Fix} B_{n}=\left\{\lim _{j \rightarrow \infty} B_{n}^{j} \varphi\right\} .
$$

Since $e_{0}, e_{1} \in \operatorname{Fix} B_{n}$, where $e_{i}(t):=t^{i}$ for $i=0,1$ and $t \in[0,1]$, and Fix $B_{n}$ is a linear subspace of $X$, we infer the function $t \mapsto \varphi(0)(1-t)+\varphi(1) t$ belongs to Fix $B_{n} \cap\left(\varphi+X_{0}\right)$. Hence and by (4.2), we get (4.1).

Remark 4.1. The above arguments show that each Bernstein operator $B_{n}$ is a Banach $G$-contraction satisfying the assumptions of Theorems 3.2, 3.3 and 3.4. Also, Theorem 3.5 is applicable here because it is easily seen that $B_{n}$ is nonexpansive. On the other hand, since $E(G)$ is not a partial order, none of results from [RR04], [NR-L05], [PR06], [NR-L07] and [NPR-L07] can be applied here. The same remark concerns Corollary 3.4: here $E(G)$ defined in its proof is neither transitive nor weakly antisymmetric.

\section{ACKNOWLEDGMENT}

The author is grateful to Professor Juan J. Nieto for providing files of his papers.

\section{REFERENCES}

[DS58] N. Dunford and J. T. Schwartz, Linear Operators. I. General Theory, Interscience Publishers, Ltd., London, 1958. MR0117523 (22:8302)

[E61] M. Edelstein, An extension of Banach's contraction principle, Proc. Amer. Math. Soc. 12 (1961), 7-10. MR0120625 (22:11375)

[G-BL06] T. Gnana Bhaskar and V. Lakshmikantham, Fixed point theorems in partially ordered metric spaces and applications, Nonlinear Anal. 65 (2006), 1379-1393. MR2245511 (2007c:47067)

[GD03] A. Granas and J. Dugundji, Fixed Point Theory, Springer-Verlag, New York, 2003. MR1987179 (2004d:58012) 
[Ja01] J. Jachymski, Order-theoretic aspects of metric fixed point theory, Handbook of Metric Fixed Point Theory (eds., W. A. Kirk and B. Sims), 613-641, Kluwer Acad. Publ., Dordrecht, 2001. MR1904289 (2003f:54094)

[Jo97] R. Johnsonbaugh, Discrete Mathematics, Prentice-Hall, Inc., New Jersey, 1997.

[KR67] R. P. Kelisky and T. J. Rivlin, Iterates of Bernstein polynomials, Pacific J. Math. 21 (1967), 511-520. MR0212457 (35:3328)

[L95] A. Lasota, From fractals to stochastic differential equations, Chaos-the interplay between stochastic and deterministic behaviour (Karpacz, 1995), 235-255, Lecture Notes in Phys. 457, Springer, Berlin, 1995. MR1452617 (98j:28005)

[NPR-L07] J. J. Nieto, R. L. Pouso and R. Rodríguez-López, Fixed point theorems in ordered abstract spaces, Proc. Amer. Math. Soc. 135 (2007), 2505-2517. MR2302571

[NR-L05] J. J. Nieto and R. Rodríguez-López, Contractive mapping theorems in partially ordered sets and applications to ordinary differential equations, Order 22 (2005), 223239. MR2212687 (2006m:47097)

[NR-L07] J. J. Nieto and R. Rodríguez-López, Existence and uniqueness of fixed point in partially ordered sets and applications to ordinary differential equations, Acta Math. Sinica, English Ser. (2007), 2205-2212.

[PR06] A. Petruşel and I. A. Rus, Fixed point theorems in ordered L-spaces, Proc. Amer. Math. Soc. 134 (2006), 411-418. MR2176009 (2006g:47097)

[RR04] A. C. M. Ran and M. C. B. Reurings, A fixed point theorem in partially ordered sets and some applications to matrix equations, Proc. Amer. Math. Soc. 132 (2004), 1435-1443. MR2053350 (2005a:47112)

[R04] I. A. Rus, Iterates of Bernstein operators, via contraction principle, J. Math. Anal. Appl. 292 (2004), 259-261. MR2050229 (2005c:41034)

Institute of Mathematics, Technical University of Łódź, Wólczańska 215, 93-005 ŁÓDŹ, POLAND

E-mail address: jachym@p.lodz.pl 